\title{
An oblique asymptote of functions in polar coordinate system
}

\author{
Nauryzbayeva R.M. \\ Border Service Academy of National Security Committee of the Republic of Kazakhstan
}

How to cite this paper: Nauryzbayeva R.M. (2020) An oblique asymptote of functions in polar coordinate system. Journal of Applied Mathematics and Computation, 4(1), 1-4.

DOI: $\underline{10.26855 / \mathrm{jamc} .2020 .03 .001}$

Received: May 23, 2019

Accepted: June 20, 2019

Published: February 19, 2020

*Corresponding author: Nauryzbayeva R.M. Border Service Academy of National Security Committee of the Republic of Kazakhstan.

\begin{abstract}
In the XVII century, some scientists showed the response of studying the curves by the analytical method. As a disadvantage of this method, it was noted that its use does not reveal the natural origin of the curve, since the object of the study is actually not the curve itself, but the related equation. In this work, we consider a curve as a function given in the polar coordinate system, if its equation can be represented in an explicit form and some features of an oblique asymptote of the graph of functions.
\end{abstract}

\section{Keywords}

Radius Function, Polar Coordinates, Curves, Asymptotes

\section{Introduction}

There were many opinions after the discovery of variables on the study of curves. The criticism of the analytical method for studying the shape and properties of curves was based on the fact, that there is no visual image of this curve and geometric constructions disappear in using this method. It was indicated that the coordinate system is an extraneous element of the study, with which the curve is artificially connected.

The equations of the curves are so complex that the study of each turned into a difficult research. From history it is known, that even to identify the domain of the equation of a curve, called "the folium of Descartes", took almost half a century.

Two types of polar coordinates are defined at the moment:

1) polar coordinates, where, $p \geq 0$,

2) generalized polar coordinate system, where both, $p \geq 0$, and $p \leq 0$.

The first graph of functions is a two-sided petal flower (Fig. 1), and the second is a four-sided petal flower (Fig. 2).

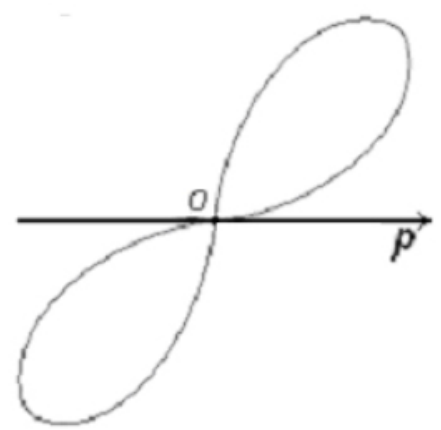

Fig. 1 polar coordinates, where, $p \geq 0$;

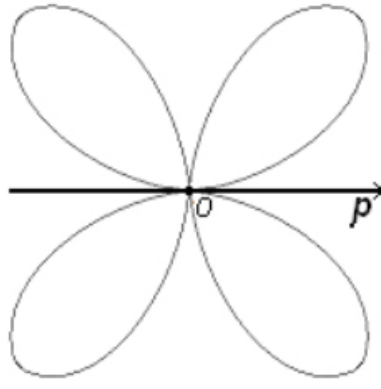

Fig. 2 polar coordinate system, where both, $\mathbf{p} \geq 0$, and $\mathbf{p} \leq \mathbf{0}$. 
We study the first type of polar coordinates. If there is a curve equation $p=p(\varphi)$ in the polar coordinate system, then it can be considered as $\varphi-$ a function of the variable, where $0 \leq \varphi<\infty$. Finding the domain of a function should be identified from the solution of inequality $p \geq 0$.

Finding asymptotes is one of the key stages in the study of functions. Work [1] defines 4 types of asymptotes of functions given in the polar coordinate system and the formulas for finding them, if they exist.

1) Oblique asymptote. It is known that the oblique asymptote has the following equations in the Cartesian and polar coordinate systems:

$$
y=k x+b \text { and } p=\frac{b}{\sin \varphi+k \cos \varphi}
$$

In the polar coordinate system, the oblique asymptote should be found at the points, where one of the limits , $\lim _{\varphi \rightarrow \varphi_{0}-0} p$, $\lim _{\varphi \rightarrow \varphi_{0}-0} p$ goes to infinity and coefficients are found by the formula:

$$
k=\lim _{\varphi \rightarrow \varphi 0} \frac{\sin \varphi}{\cos \varphi}, b=\lim _{\varphi \rightarrow \varphi 0}(p \sin \varphi-k p \cos \varphi)
$$

2) Horizontal asymptote. This will be defined in the points, where one of the limits is $\lim _{\varphi \rightarrow \varphi_{-}} p \lim _{\varphi \rightarrow \varphi_{+}} p \lim _{\varphi \rightarrow \pi^{-}} p \lim _{\varphi \rightarrow \pi^{+}} p$ goes to infinity. In these values $-k=\lim _{\varphi \rightarrow \varphi 0} \frac{\sin \varphi}{\cos \varphi}=0$. If one of the limits is $\lim _{\varphi \rightarrow \varphi_{-}} p \sin \varphi, \lim _{\varphi \rightarrow \varphi_{+}} p \sin \varphi, \lim _{\varphi \rightarrow \pi^{-}} p \sin \varphi, \lim _{\varphi \rightarrow \pi^{+}} p \sin \varphi$, is a finite number and is $b$, then there is the equation of a horizontal asymptote is $p=\frac{b}{\sin \varphi}$.

Note 2. The polar coordinate system does not limit the horizontal asymptote to the radius function at infinity (Fig. 3).

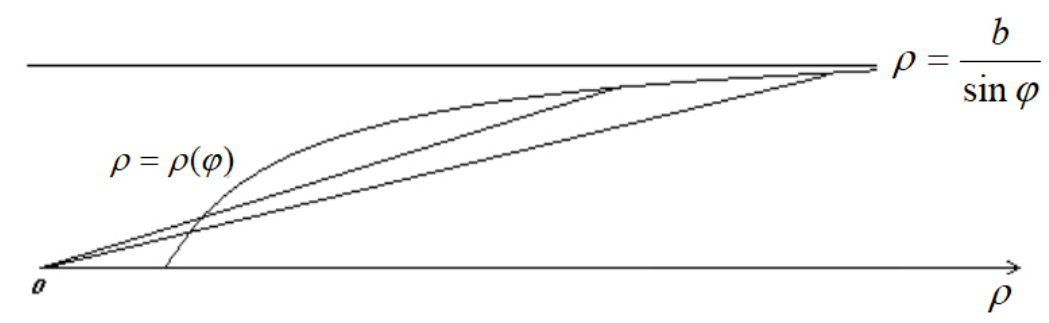

Fig. 3 horizontal asymptote of the curve

3) Vertical asymptote. If one of the limits is $\lim _{\varphi \rightarrow \frac{\pi}{2}-0} p \lim _{\varphi \rightarrow \frac{\pi}{2}+0} p \lim _{\varphi \rightarrow \frac{2 \pi}{2}-0} p \lim _{\varphi \rightarrow \frac{2 \pi}{2}+0} p$ goes to $\infty$ and one of the limits goes to $\lim _{\varphi \rightarrow \frac{\pi}{2}-0} p \cos \varphi, \lim _{\varphi \rightarrow \frac{\pi}{2}-0} p \cos \varphi, \lim _{\varphi \rightarrow \frac{2 \pi}{2}-0} p \cos \varphi, \lim _{\varphi \rightarrow \frac{2 \pi}{2}+0} p \cos \varphi$ goes to a constant $b$, then $p=\frac{b}{\cos \varphi}$ is a vertical asymptote.

4) Circular asymptote. If $\lim _{\varphi \rightarrow \infty} p(\varphi)$ is a finite number $r$ for this curve, then there is $p=r$ is a circular asymptote for this curve. [2].

In this paper, we define some features of the oblique asymptote of functions given in the polar coordinate system. For example:

Identify an asymptote of the graph of functions by the equation of $p=\frac{\varphi}{\varphi-1}$.

Solution. Firstly, in $\varphi \rightarrow \infty$ radius function approaches 1 . Then, from the definition it follows that $p=1$ is the circular asymptote (Figure 4).

\section{Methods}

All asymptotes, except circular, we will define in the values of $\varphi$ argument, where radius function is $p \rightarrow \infty$. Here, we see that in $\varphi \rightarrow 1$ radius function $p \rightarrow \infty$. Then, from the formulas (1) we get:

$$
k=\lim _{\varphi \rightarrow 1} \operatorname{tg} \varphi=\operatorname{tg} 1,
$$


$b=\lim _{\varphi \rightarrow 1} p(\sin \varphi-\operatorname{tg} 1 \cdot \cos \varphi)=\lim _{\varphi \rightarrow 1} \frac{\varphi(\sin \varphi-\operatorname{tg} 1 \cdot \cos \varphi)}{\varphi-1}=\lim _{\varphi \rightarrow 1} \frac{(\sin \varphi-\operatorname{tg} 1 \cdot \cos \varphi)+\varphi(\cos \varphi-\operatorname{tg} 1 \cdot \sin \varphi)}{1}=\frac{\cos ^{2} \varphi+\sin 1 \sin \varphi}{\cos \varphi}=\frac{1}{\cos 1}$

Then the oblique asymptote in the Cartesian and polar coordinate systems, respectively, has the form:

In polar coordinates we get:

$$
y=\operatorname{tg} 1 \cdot x+\frac{1}{\cos 1},
$$

$$
p=\frac{1}{\cos 1(\sin \varphi-\operatorname{tg} 1 \cos \varphi)}, \text { or } p=\frac{1}{\sin (\varphi-1)} \text {. }
$$

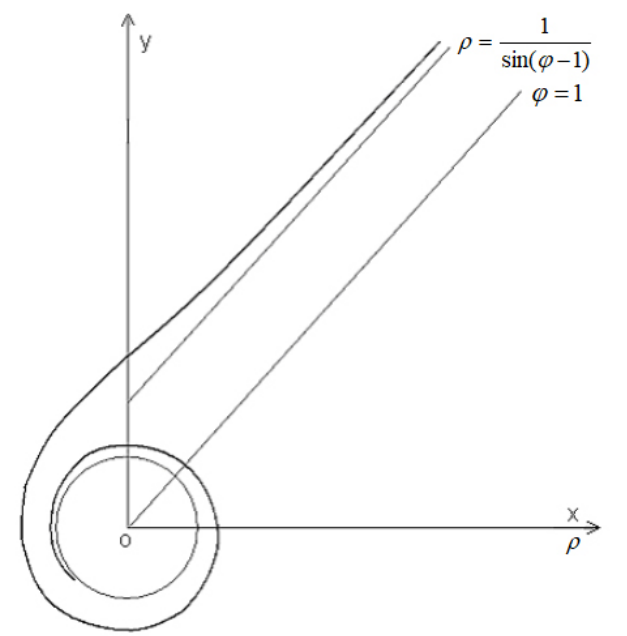

Fig. $4-$ Curve $p=\frac{\varphi}{\varphi-1}$ and its asymptote $p=\frac{1}{\sin (\varphi-1)}$.

Let's make the table of values of the given functions and its inclined asymptotes. In the segment $(1,+\infty)$

From the table we see, that with approaches of an argument to the angle $\varphi=1$, where function tends to infinity, an absolute value of radius function and asymptote approaches 1.

Table 1 Group.

\begin{tabular}{cccccccc}
\hline$\varphi^{0}$ & 900 & 600 & 59,590 & 58,440 & 57,880 & 57,750 & 57,350 \\
\hline р-радиан & 1,57 & 1,05 & 1,04 & 1,02 & 1,01 & 1,008 & 1,001 \\
\hline$\frac{\varphi}{\varphi-1}$ & 2,75 & 21 & 26 & 51 & 101 & 126 & 1001 \\
\hline$\frac{1}{\sin (\varphi-1)}$ & 1,85 & 20,008 & 25,007 & 50.0033 & 100,002 & 125,001 & 1000,0001 \\
\hline$\left|p^{*}-p\right|$ & 1,1 & 0,992 & 0,993 & 0,9967 & 0,998 & 0,999 & 0,9999 \\
\hline
\end{tabular}

\section{Results}

And also by finding the following limit, we see that the difference between the function and the asymptote is:

$$
m=\lim _{\varphi \rightarrow 1}\left(\frac{\varphi}{\varphi-1}-\frac{1}{\sin (\varphi-1)}\right)=\lim _{\varphi \rightarrow 1} \frac{(\varphi \sin (\varphi-1)-(\varphi-1))}{((\varphi-1) \sin (\varphi-1))}=\lim _{\varphi \rightarrow 1} \frac{\sin (\varphi-1)+\varphi \cos (\varphi-1)-1}{\sin (\varphi-1)+(\varphi-1) \cos (\varphi-1)}=\lim _{\varphi \rightarrow 1} \frac{2 \cos (\varphi-1)-\varphi \sin (\varphi-1)-1}{2 \cos (\varphi-1)-(\varphi-1) \sin (\varphi-1)}=1
$$

\section{Discussion}

This geometric fact can be interpreted as follows. 
If we indicate $\alpha_{i}=<A_{i} B_{i} C_{i}=\varphi_{i}-\varphi_{0}, i=1, \ldots, n$ then in $\varphi \rightarrow \varphi_{0}$ the sequence of angles $\alpha_{1}, \alpha_{2}, \ldots, \alpha_{n}$ approach zero in $n \rightarrow \infty$ Then, in rectangle $\triangle B_{i} C_{i} A_{i}$ catheti $d_{i}=l_{i} \sin \alpha_{i}, i=1, \ldots, n$ are the length of a perpendicular falling from the points $B_{1}$, $B_{2}, \ldots, B_{n}$ to an asymptote.

$d_{i}$ can approach to zero, while $l_{i}$ - can stay constant, i.e. $l_{1}=l_{2}=\ldots=l_{n}$, in $n \rightarrow \infty$. $d_{i} \rightarrow 0$, because $\alpha_{i} \rightarrow 0$, so as $\varphi \rightarrow \varphi_{0}$ (Fig. 5)

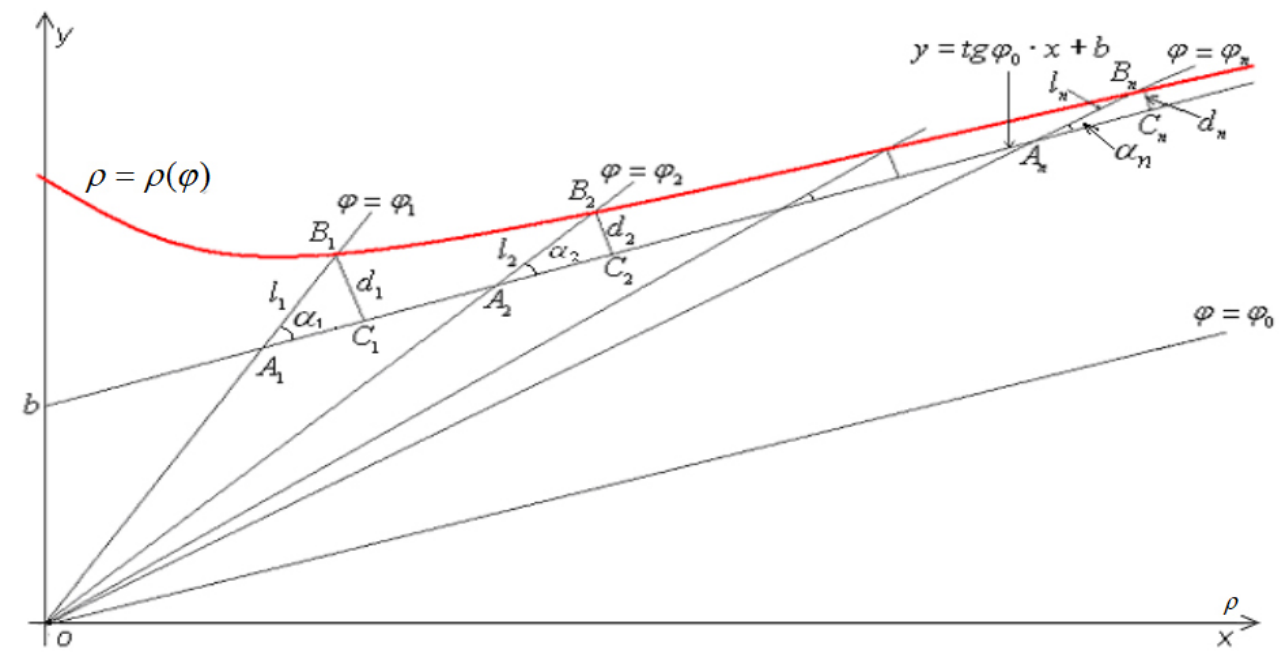

Fig.5 - Radius function of a curve and radius function of an asymptote in $\varphi \rightarrow \varphi_{0}, d_{i} \rightarrow 0$ can be related by.

\section{Conclusion}

Radius function of a curve and radius function of an asymptote incan be related by

$$
\lim _{\varphi \rightarrow \varphi_{0}}\left|p-\frac{b}{\sin \varphi-k \cos \varphi}\right|=m,(m \geq 0, m-\text { const })
$$

\section{Acknowledgements}

As a result, we obtained that at infinity, the asymptote and the curve approach each other, but keep the constant difference of the radii of the functions and asymptote.

\section{References}

[1] Nauruzbayeva R.M. ALGORITHM OF FINDING EXTREME POINTS OF CURVES. The conference held on 1112-13 May 2018 at the branch campus of KBU in a UNESCO World Heritage City; SAFRANBOLU http://icatces. org/ and be indexed on http://indexive.com.

[2] Nauruzbayeva R.M. Condition of bulge and concavity of a curve in the arctic system of coordinates in the extreme points of radius of functions Extended Abstracts of the 6th Seminar in Geometry and Topology Bonab, East Azerbaijan, Iran, 18-20 September 2011.-239 c. 